\title{
Massive haematometrosalpinx in a young girl with cloacal anomalies: Laparoscopic management
}

\author{
D Silva', P Randombage ${ }^{\mathrm{b}}$, W Gankanda ${ }^{\mathrm{b}}$, J Mathanamohanc, S Abeygunasekera ${ }^{\mathrm{d}}$
}

\begin{abstract}
A cloacal anomaly refers to the collection of defects that occur during fetal development, where the intestinal, urinary and reproductive tracts open into a common cavity. It is imperative to address this condition since despite its fairly low incidence and limited data, it has a very high impact on the patient's quality of life, and therefore mandates further study to effectively manage these patients. This study is regarding a 13-year-old girl diagnosed to have a cloacal anomaly since birth presenting with cyclical abdominal and pelvic pain for 6 months. She had undergone multiple abdominal and pelvic corrective surgeries since birth, leading to a colostomy and Mitrofanoff channel for bowel and bladder control, respectively. Diagnostic imaging revealed bilateral haematosalpinx for which ultrasound guided aspiration followed by subcutaneous administration of $\mathrm{GnRH}$ analogues was given as a temporary mode of relief which eventually recurred within 4 months. Following an extensive Multidisciplinary discussion, the team proceeded with the crucial decision of a hysterectomy and bilateral salpingectomy. The surgery itself was challenging due to multiple adhesions, scarring and difficulty in port placement. Following surgery, the patient's quality of life significantly improved allowing her to attend to her activities of daily living and maintain a social life. The need for future appointments to address sexual functions and fertility wishes was explained.
\end{abstract}

\section{Introduction}

\section{What are cloacal anomalies}

A cloaca is the persistence of a common cavity into which the intestinal, urinary and reproductive tracts open. Cloacal exstrophy (CE) is a devastating condition that appears to be the result of a very early developmental defect of the caudal eminence ${ }^{1}$. This anomaly occurs as a result of incomplete descent of the urorectal septum, which fails to reach the perineum, and the associated maldevelopment of the mullerian tubercle, sino- vaginal bulbs, vaginal plate and urogenital sinus ${ }^{2}$.

These patients typically present with an omphalocele, imperforate anus with a shortened hind gut, and an

Sri Lanka Journal of Obstetrics and Gynaecology 2021; 43: 197-202

DOI: http://doi.org/10.4038/sljog.v43i3.7990

a Senior Lecturer, Department of Obstetrics and Gynaecology, Faculty of Medical Sciences, University of Sri Jayewardenepura, Sri Lanka

${ }^{\mathrm{b}}$ Senior Registrar in Obstetrics and Gynaecology, Colombo South Teaching Hospital, Kalubowila, Sri Lanka

${ }^{c}$ House Officer in Obstetrics and Gynaecology, Colombo South Teaching Hospital, Kalubowila, Sri Lanka

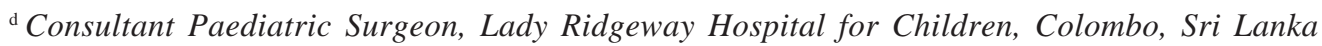

Correspondence: PR, e-mail: prabathrandombage@gmail.com

D https://orcid.org/0000-0002-3576-9923

Received $4^{\text {th }}$ April 2021

Accepted $11^{\text {th }}$ October 2021

This is an open-access article distributed under the terms of the Creative Commons Attribution 4.0 International License, which permits unrestricted use, distribution and reproduction in any medium provided the original author and source are credited. 
open ceacal plate flanked by open hemibladders on either side. In addition many patients also have deformities of the spinal cord, kidneys and ureters ${ }^{1}$.

However, cloacal exstrophy (CE) is a rare birth defect, occurring in 1:200,000 to $1: 400,000$ births $^{3}$ and accounts for about $10 \%$ of all anorectal malformations in females ${ }^{4}$.

Managing patients with a cloacal anomaly since childhood, mainly during puberty and addressing their sexual and reproductive needs remains a difficult reconstructive challenge as data on long term outcomes are scarce.

\section{Obstetric, gynaecological and social impact}

Gynaecological abnormalities are common with the cloaca due to their abnormal uterine anatomy and various invasive gynaecological procedures but may remain asymptomatic until puberty or adult life ${ }^{2}$. They have normal ovaries but owing to uterine anomalies their Mullerian ducts do not fuse, resulting in uterine and vaginal duplication. Surgeons have performed various operations ranging from ablation of the septum to removal of one of the two halves of the uterus/ hemi-hysterectomy to total hysterectomy. If the uterus is left in situ, complications such as uterine prolapse and hydro- or hematometrocolpos and haematosalpinx may occur which warrant further interventions.

They have difficulty in becoming pregnant, and if they do conceive, they are unlikely to carry the pregnancy to term ${ }^{1}$. Among patients with isolated reproductive malformations, the rate of caesarean section is higher secondary to dystocia and foetal malpresentation; in the population of persistent cloaca, elective caesarean section is recommended to retain the previous reconstructive procedures ${ }^{3}$.

Patients with persistent cloaca invariably require numerous operations and lengthy hospitalizations leading to severe morbidity with poor quality of life ${ }^{5}$. New parents of daughters born with this anomaly should be counselled about future genital reconstruction. Counselling is challenging due to the paucity of data, hence future research into sexual activity, satisfaction with genital reconstruction, and reproductive outcome is warranted.

We hope to add data to literature regarding the crucial decision of performing a hysterectomy and bilateral salpingectomy of a young patient with persistent cloaca, who has had multiple surgeries in childhood which have resulted in abdominal and severe perineal scarring. In addition, this case report will address the best route of surgery, important aspects of laparoscopy such as port placement, and the need for experts to perform such procedures in the background of past multiple surgeries.

\section{Case history}

A 13-year-old schoolgirl was referred to us by the paediatric surgical team due to severe cyclical abdominal and pelvic pain over 6 months duration. She was a diagnosed patient with a cloacal anomaly at birth. She had exomphalos, an imperforated anus and a patent urachus, for which she had undergone exploratory laparotomy and formation of a colostomy and vesicostomy at day one of birth. At one month of age she developed intestinal obstruction and underwent a right transverse exploratory laparotomy to release ischemic bowel, and bowel anastomosis and a formation of a dependent vesicostomy. Since then, she developed 3 episodes of intestinal obstruction which were managed conservatively.

At the age of 1 year and 4 months, she underwent correction of cloacal exstrophy where the vaginal component of the cloaca was separated from the bladder, and the vaginal orifice was reconstructed using peritoneum. At the same time, the rectum was separated and brought down. Since then, she had multiple cases of EUA, V-Y anoplasty and candle dilatation of the anus till she reached the age of 4 years. Following this, she soon developed haematuria, and underwent cystoscopy where she was found to have a large patulous opening which led into the urethra with a continuous leak. By the age of 7 years, due to persistent urinary leak and recurrent urinary tract infections, she underwent creation of a Mitrofanoff channel, where the appendix was mobilized and anastomosed between the bladder and right sided abdominal wall. Her perineal bladder opening was closed, and she was taught selfcatheterization. Since then she had another 4 surgeries for refashioning and dilatation of the Mitrofanoff. Her perineal rectal and vaginal openings were closed by then and the colostomy was functioning well.

As she reached the age of 13 , she developed cyclical pelvic and abdominal pain, scoring 8/10. She had her thelarche and adrenarche by then. The pain was substantial enough for her to refrain from schooling 
and affected her activities of daily living significantly. USS, CECT and MRI revealed massive haemato-metrosalphinx. She underwent ultrasound guided aspiration to alleviate her symptoms which was followed by 3 cycles of monthly injections of subcutaneous $\mathrm{GnRH}$ analogues.

Four months later, she presented with the same constellation of symptoms, and a repeat MRI scan reconfirmed the persistence of bilateral haematosalpinx and a haematometron. Following an extensive multidisciplinary team discussion involving a gynaecologist, a peadiatric surgeon, a radiologist and a psychiatrist, the crucial decision for a hysterectomy and bilateral salpingectomy was made. This intricate surgery was performed at the Professorial unit of Obstetrics and Gynaecology, Colombo South Teaching Hospital, Kalubowila.

Palmer's point entry was made, followed by a paramedian $10 \mathrm{~mm}$ medial port and 2 customized 5 mm lateral ports (Figure 1). A large right sided haematosalpinx extending up to the undersurface of the liver and a left sided haematosalpinx buried in deep bowel adhesions were identified intra-operatively (Figure 2). The uterus was found to be displaced caudally towards the RIF region, with a rudimentary cervix lacking vaginal continuation. Meticulous laparoscopic adhesiolysis, total hysterectomy and bilateral salpingectomy was done. Tissues were retrieved via the lateral port by morcellation due to severe scarring of the midline abdominal wall. Her colostomy and Mitrofanoff tracts were preserved.

ICU care was provided for a period of 2 days, following which she was discharged at the $4^{\text {th }}$ post-operative day. Three doses of monthly GNRH analogues were then given to regress any residual endometriotic tissue. Her pain score, schooling and quality of life drastically improved following surgery. Furthermore, future appointments until she reached adulthood were arranged, where creation of a neovagina, future sexual functions and fertility options from her own eggs is to be discussed.

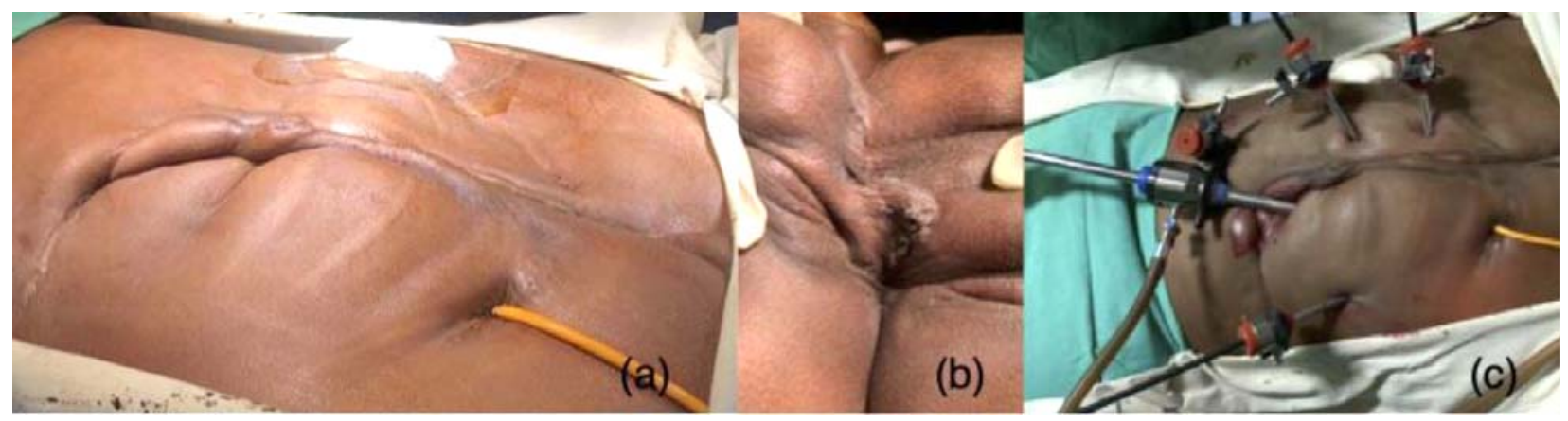

Figure 1. Abdominal (a), perineal scaring (b) and port placement (c).

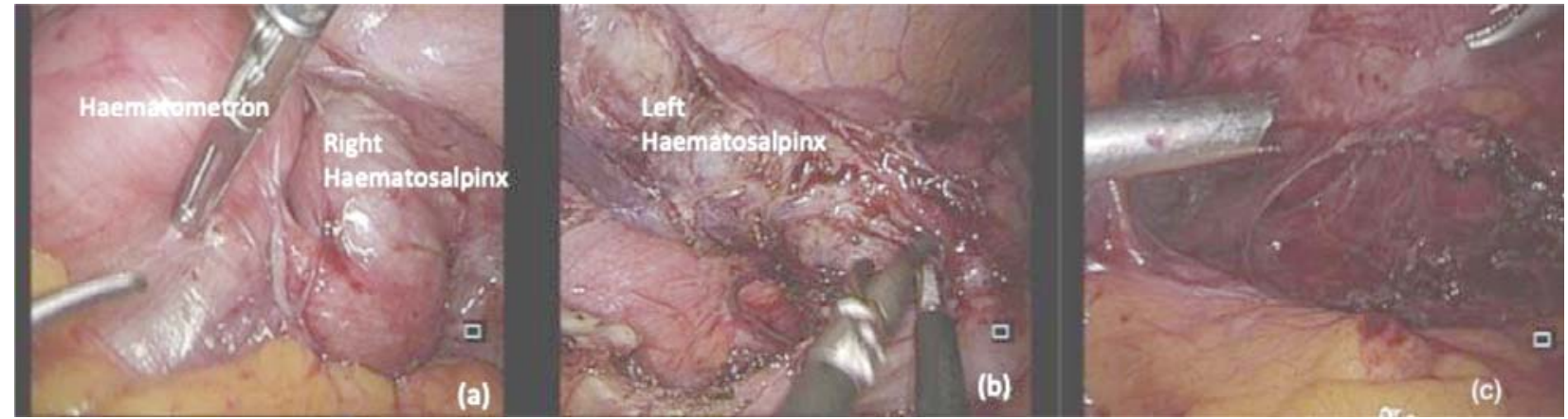

Figure 2. Right (a) and left haematosalpinx (b) and final surgical outcome (c). 


\section{Discussion}

\section{Means of managing gynaecological issues}

The principal objectives of surgical procedures are the establishment of bowel and bladder control, as well as normal sexual function. However, many of these girls need additional surgical procedures not only to achieve continence, but also to allow normal menstruation and sexual activity ${ }^{2}$.

Hendren advocated the simultaneous repair of the rectal and urogenital components, which was performed predominantly by an abdominoperineal pull-through in his early series ${ }^{4}$. Since 1989 Penã has advocated the treatment of cloaca by a posterior sagittal anorectovagino-urethroplasty ${ }^{6}$. In this occasion the vagina may have to be brought down to the perineum, and if that is not feasible bowel could be interposed to create a neovagina ${ }^{7}$.

All patients with the exstrophy-epispadias complex should undergo renal US at birth. Concomitant pelvic US should be considered if the abdominal wall defect allows it. Careful intraoperative evaluation at the time of omphalocele and bladder closure should be performed ${ }^{3}$.

Due to the high incidence of problems seen at puberty it is necessary to reassess all of these girls in the peripubertal period. Ultrasound of the pelvis is useful for screening, whereas MRI is the investigation of choice to document the complex anatomy in these patients particularly when reconstructive surgery is necessary. Fedele et al. reported 100\% sensitivity and $79 \%$ specificity in the diagnosis of congenital uterine anomalies $^{8}$.

Examination with the patient under anesthesia and vaginoscopy are also recommended to assess vaginal patency, adequacy and the presence of a cervix (cervices). Findings previously documented in infancy may be misleading ${ }^{2}$. However, in our case as there is no patent vaginal orifice, vaginoscopy and EUA are not applicable.

In the case of a long common cloacal channel, additional manoeuvres such as labial or perineal skin flaps, tubularization of a vaginal flap or vaginal replacement with intestine, may be necessary. We believe it is appropriate to avoid ablative genital surgery as far as possible. In view of the many advances being made in the field of fertility, detailed discussion with physicians experienced in the care is vital for these patients to become pregnant ${ }^{1}$.

Trying to retain at least part of their uterus with a low rate of complications (hemi-hysterectomy) and attempts at retaining all or part of the uterus seem justified unless the anatomy is grossly abnormal. Such an approach gives these patients a sense of normality and permits them to relate to their peers as they mature. In addition, they retain the potential to bear children. However, in severe cases like the case being discussed, a total hysterectomy should be offered after a detailed multidisciplinary discussion to avoid constant agony throughout her reproductive life, and to maintain bowel and urethral continence which are more important aspects of the patient's quality of life.

After initial reconstruction, patients might have multiple additional surgeries for stoma reversal, bladder augmentation, and creation of conduits, all with high potential for pelvic adhesions. Postpubertal adolescents who had no problems at menarche may yet require revision surgery for cosmetic reasons or to facilitate sexual intercourse for vaginal stenosis as young adults².

Data regarding pregnancy, fertility rates, mode of delivery, and obstetric outcomes are limited in this patient population, making it challenging to counsel patients and their families. It is imperative to note that patients with repaired cloacal malformations are at an increased risk of preterm birth and cesarean delivery ${ }^{9}$.

\section{Justification for total hysterectomy}

Gynecological surgery in these patients is evidently difficult and many patients require future revisions and reconstructions. Hence the implication of salvaging the native female internal genital tract has been questioned $^{10}$.

Considering adult gynecological literature, mullerian abnormalities such as rudimentary cornua, uterus didelphys and vaginal atresia can lead to obstruction of menstrual outflow resulting in hematocolpos, hematometra, hematosalpinx and endometriosis from menstrual blood filling these structures.

In a study conducted among 41 patients, all were evaluated at puberty, and 28 (68\%) had uterine function, 13 (32\%) were menstruating normally and 15 (36\%) presented with hematometra/hematocolpos. All 15 girls with an obstructed uterus required surgery, which 
included hysterectomy in 2, partial hysterectomy with vaginoplasty in 3 and vaginoplasty in $9^{2}$. In our case she had already started retrograde menstruation giving rise to severe pelvic pain which can potentially persist all through her reproductive life. In addition, the significant abdominal and perineal scarring also mandate a permanent solution.

Levitt et al also reported an obstructed uterus in $41 \%$ of their 22 cloaca cases. All of their patients who presented as teenagers with cystic abdominal masses underwent surgical resection of the cystic masses involving the fallopian tubes and uteri ${ }^{11}$.

Pregnancy is possible in female patients with persistent cloaca, but it carries a high risk for the patient. Mathews et al described a patient with persistent cloaca who delivered a child by caesarean section but was later complicated by perforation of the Koch pouch and post-partum uterine prolapse. She also started leaking stool and her urinary stoma became incontinent ${ }^{12}$. It seems prudent to recommend that these patients only become pregnant after a detailed and careful discussion with an experienced high-risk obstetrician and the surgeon coordinating her care. Taking this case with severe abdominal and pelvic scarring into account, attempts at retaining fertility may come with a price of bowel and urethral damage.

Creation of a neovagina merely to have sexual functions in the background of compromised fertility is a topic of debate. In a study conducted among 21 older girls (age at review 17 to 32 years, mean 24) 12 have been sexually active and 6 have been examined by a gynaecologist and have an adequate vagina but are not sexually active. Of them 4 patients have required revision vaginal surgery in adulthood to facilitate intercourse (re-do vaginoplasty in 3, introitoplasty in 1). There have been no pregnancies in this series to date $^{2}$. In our case, authors believe that creation of a neovagina is surgically quite challenging due to significant perineal scarring. However, it is open to discussion when she contemplates the start of her sexual life.

Some studies described alternative medical treatments such as with anovulatory drugs (luteinizing hormonereleasing hormone analogues) and delayed repair involving vaginoplasty and/or division of vaginal septae preserving uterine structures when possible ${ }^{2}$. However, long term medical treatments lack sufficient evidence in the background of severe cases of scarring where reconstruction is not practical like our case. In addition, long term medical treatment has its own set of complications, including medical menopause.

Timing of surgery is also very important in patients with cloacal anomalies. Bowel, bladder and urethral functions are usually restored in childhood. The decision to preserve or remove the uterus and reconstruction surgeries to retain sexual functions are to be done during the peripubertal period or even later in life. However, when reconstruction was performed prior to puberty, secondary procedures were usually small and well tolerated. In a similar study which was conducted, the average age for patients undergoing their first mullerian procedure was just under 12 years, and pre-pubertal which is similar to our case ${ }^{3}$.

\section{Laparoscopy as the best route of surgery}

Laparoscopy is a useful diagnostic tool, and has the added advantage of being a modality of treatment simultaneously ${ }^{13}$. In recent years, laparoscopy has become a viable alternative to laparotomy for the management of uterine congenital anomalies.

Commonly accepted benefits of the method, such as improved visualization of the pouch of Douglas, smaller incisions, decreased adhesion formation, reduced postoperative pain and shorter hospital stay, are in favour of a laparoscopic approach ${ }^{13}$. However, other factors such as availability of suitable instruments and surgical expertise need to be considered.

Conventional methods for port placement in gynaecological laparoscopy, where intraperitoneal adhesions are anticipated have been described, such as the palmer's point, the $10 \mathrm{~mm}$ port in the midline and the lateral ports below it. Our case, however, was complicated with multiple laparotomy scars, a colostomy on the left and a ureterostomy on the right, being the common sites used by gynaecologists to insert lateral ports to access the pelvis. We started with a palmer's point entry, followed by the main port placement on the right paramedian area securing the colostomy (Figure 1). Other lateral ports were placed under direct vision where space was provided. Considering the fact that teenagers have a limited abdominal wall area when compared to adults, the surgery needs to be carefully pre-planned with the aid of MRI images, and port placement needs to be methodically carried out with careful precision by an experienced gynaecologist. 


\section{Conclusion}

This case emphasizes on the importance of a multidisciplinary team to come to a decision as crucial as a hysterectomy and bilateral salpingectomy for a teenage girl with persistent cloaca having bilateral haematosalpinx and haematometron as a result. Bearing in mind the multiple abdominal and pelvic surgeries she had undergone over 13 years leading to severe adhesions and scarring, medical management to alleviate her symptoms is not feasible. Instead, a surgical approach using laparoscopy that acts as a diagnostic as well as therapeutic tool is the best mode of treatment. Symptomatic relief is only the beginning of the treatment cascade of this condition. The need for sexual functions and fertility wishes need to be addressed with time, and appropriate interventions need to be done to further improve the patient's quality of life and wellbeing.

\section{Declaration}

Conflicts of interest: There are no conflicts of interest.

\section{Acknowledgements}

We would like to extend our gratitude to the Director, and theatre staff of Operation theatre B, Colombo South Teaching Hospital, Kalubowila, Sri Lanka for their support in performing this complex surgery. We are grateful to Prof Thejana Wijeratne, Consultant Surgeon, Colombo South Teaching Hospital, Kalubowila, Sri Lanka.

\section{References}

1. Naiditch JA, Radhakrishnan J, Chin AC, Cheng E, Yerkes E, Reynolds M. Fate of the uterus in 46XX cloacal exstrophy patients. J Pediatr Surg [Internet]. 2013; 48(10): 2043-6. Available from: http://dx.doi.org/10.1016/j.jpedsurg.2013.02.040

2. Warne SA, Wilcox DT, Creighton S, Ransley PG. Long-term gynecological outcome of patients with persistent cloaca. J Urol. 2003; 170(4 II): 1493-6.

3. Suson KD, Preece J, Di Carlo HN, Baradaran N, Gearhart JP. Complexities of Müllerian Anatomy in 46XX Cloacal Exstrophy Patients. J Pediatr Adolesc Gynecol [Internet]. 2016;29(5):424-8. Available from: http://dx.doi.org/10.1016/ j.jpag.2016.01.124

4. Hendren WH. Cloaca, the most severe degree of imperforate anus: experience with 195 cases. Ann Surg. 1998; 228(3): 331.

5. Molenaar JC. Cloacal exstrophy. In: Seminars in pediatric surgery. 1996. p. 133.

6. Peña A. The surgical management of persistent cloaca: results in 54 patients treated with a posterior sagittal approach. J Pediatr Surg. 1989; 24(6): 590-8.

7. Radhakrishnan J. Double-barrelled colovaginoplasty in a patient with cloacal exstrophy variant. J Pediatr Surg. 1998; 33(9): 1402-3.

8. Fedele L, Dorta M, Brioschi D, Massari C, Candiani GB. Magnetic resonance evaluation of double uteri. Obstet Gynecol. 1989; 74(6): 844-7.

9. Vilanova-Sanchez A, McCracken K, Halleran DR, Wood RJ, Reck-Burneo CA, Levitt MA, et al. Obstetrical Outcomes in Adult Patients Born with Complex Anorectal Malformations and Cloacal Anomalies: A Literature Review. J Pediatr Adolesc Gynecol [Internet]. 2019; 32(1): 7-14. Available from: https://doi.org/10.1016/j.jpag.2018.10.002

10. Soffer SZ, Rosen NG, Hong AR, Alexianu M, Peña A. Cloacal exstrophy: a unified management plan. J Pediatr Surg. 2000;35(6):932-7.

11. Levitt MA, Stein DM, Pena A. Gynecologic concerns in the treatment of teenagers with cloaca. J Pediatr Surg. 1998; 33(2): 188-93.

12. Mathews RI, Gan M, Gearhart JP. Urogynaecological and obstetric issues in women with the exstrophy-epispadias complex. BJU Int. 2003; 91(9): 845-9.

13. Liatsikos SA, Tsikouras P, Souftas V, Ammari A, Prassopoulos P, Maroulis G, et al. Diagnosis and laparoscopic management of a rudimentary uterine horn in a teenage girl, presenting with haematometra and severe endometriosis: Our experience and review of literature. Minim Invasive Ther Allied Technol. 2010; 19(4): 241-7. 